
легочных инфекционных осложнений на фоне острого и хронического отторжения трансплантата почки

\author{
Ф.Г. Назыров, Р.А. Ибадов, Ф.Ш. Бахритдинов, С.Х. Ибрагимов, З.Т. Маткаримов \\ ГУП «Республиканский специализированный научно-практический медицинский центр \\ хирургии им. акад. В. Вахидова», \\ 100115, Республика Узбекистан, Ташкент, Чиланзарский р-н, ул. Кичик халка йули, д. 10 \\ Контактная инорормация: Феруз Гафурович Назыров, академик РАЕН, директор Республиканского \\ специализированного научно-практического медицинского центра хирургии им. акад. В. Вахидова, \\ e-mail: dok.mzt@mail.ru \\ Дата поступления статьи: 25.01.2018 \\ Принята в печать: 21.03.2018
}

Цель: определение особенностей респираторной и интенсивной терапии пациентов с легочными инфекиионныли осложненияли на фбоне острого и хронического отторжения трансплантата почки.

Материал и методы. Ретроспективно бъли изучены результаты лечения 7 пациентов с легочныли инфекционныли осложнениями на ббоне острого и хронического отторжения трансплантата почки.

Результаты. Общее количество случаев легочных инфбекиионных осложнений составило 7 из 54 трансплантаиий почек от живъх родственных доноров. Во всех наблюдениях бъла диагностирована двусторонняя интерстициальная пневмония. Положительную динамику на боне проводимой респираторной и интенсивной терапии имели 6 пациентов.

Ключевые слова: пересадка почки, иммуносупрессия, хроническое отторжение трансплантата, инфекция, повреждение легких, интенсивная терапия

Назыров Ф.Г., Ибадов Р.А., Бахритдинов Ф.Ш. и др. Особенности респираторной и интенсивной терапии легочных инфекционных осложнений на фоне острого и хронического отторжения трансплантата почки. Трансплантология. 2018;10(1):105-109. DOI: $10.23873 / 2074-0506-2018-10-2-105-109$

\title{
Specific features of respiratory and intensive therapy for pulmonary infectious complications of acute and chronic kidney graft rejection
}

\author{
F.G. Nazyrov, R.A. Ibadov, F.Sh. Bakhritdinov, S.Kh. Ibragimov, Z.T. Matkarimov \\ Republican Specialized Scientific and Practical Medical Center of Surgery n.a. acad. V. Vakhidov \\ 10 Chilanzarskiy district, Kichik khalka yuli street, Tashkent 100115 Republic of Uzbekistan \\ Correspondence to: Feruz G. Nazyrov, Acad. of RANS, Director of Republican Specialized Scientific and Practical \\ Medical Center of Surgery n.a. acad. V. Vakhidov, e-mail: dok.mzt @mail.ru
}

Received: 25 January 2018

Accepted for publication: 21 March 2018

The purpose was to determine the specific features of respiratory and intensive therapy of pulmonary infectious complications in acute and chronic kidney graft rejection.

Material and methods. The results of treatment of 7 patients with pulmonary infectious complications against an acute and chronic kidney graft rejection were retrospectively studied.

Results. The total number of pulmonary infection cases made 7 among 54 cases of kidney transplantation from living related donors. In all cases, bilateral interstitial pneumonia was diagnosed. Six patients had positive dynamics on receiving a respiratory therapy and intensive care.

Keywords: kidney transplantation, immunosuppression, chronic transplant rejection, infection, lung injury, intensive care

Nazyrov F.G., Ibadov R.A., Bakhritdinov F.Sh., et al. Specific features of respiratory and intensive therapy for pulmonary infectious complications of acute and chronic kidney graft rejection. Transplantologiya. The Russian Journal of Transplantation. 2018;10(2):105109. (In Russian). DOI:10.23873/2074-0506-2017-10-2-105-109 
ВАП - вентилятор-ассоциированная пневмония

ИВЛ - искусственная вентиляция легких

ИПВЛ - интрапульмональная перкуссионная вентиляция легких

ОП - оптическая плотность

ОРДС - острый респираторный дистресс-синдром

\author{
ПДКВ - положительное давление в конце выдоха \\ ПЦР - полимеразная цепная реакция \\ СРБ - - -реактивный белок \\ ТП - трансплантация почки \\ ЦМВ - цитомегаловирус \\ NiCPAP - Noninvasive Constant Positive Airway Pressure
}

Введение

Среди бактериальных инфекционных осложнений у реципиентов почечного трансплантата легочные инфекции составляют от 8 до $33 \%$ и играют важную роль в структуре летальности реципиентов аллогенной почки [1-3]. В связи с этим пациенты после трансплантации почки (ТП) должны быть отнесены к группе высокого риска развития фатальных легочных инфекций [1].

Следует помнить о том, что причиной пневмонии у больных после ТП могут быть «атипичные» возбудители (легионеллы, микоплазмы, хламидии), вирусы, пневмоцисты, грибы, микобактерии туберкулеза и нетуберкулезные микобактерии. Примерно у каждого 9-го пациента развивается полимикробная легочная инфекция, а у каждого 3-4-го больного установить этиологию пневмонии не удается, несмотря на полноценное обследование [4].

Одной из сложных задач при ведении пациентов после ТП с легочными инфекционными осложнениями, на наш взгляд, представляется выбор оптимальной респираторной тактики и патогенетической терапии при развитии острого респираторного дистресс-синдрома (ОРДС). С одной стороны, появление респираторов премиум-класса с технологиями Open Lung, электроимпедансной томографии и т.д. значительно повлияло на удовлетворительные исходы при развитии ОРДС различной этиологии, с другой - пролонгированная искусственная вентиляция легких (ИВЛ) сама по себе может послужить причиной развития вентилятор-ассоциированной пневмонии (ВАП), особенно у пациентов со слабым иммунным статусом.

В этой связи применение NiCPAP (Noninvasive Constant Positive Airway Pressure) и интрапульмональной перкуссионной вентиляции легких (ИПВЛ) определено режимом механической вентиляции легких, который, согласно данным резолюции конгресса Европейского респираторного общества (ERS-2013), обладает жизнесберегающими свойствами для пациентов, нуждающихся в оказании респираторного пособия [6].

G. Riffard et M. Toussaint (2012) [5] отмечают, что ИПВЛ позволяет пациенту дышать самостоя- тельно при помощи «перкуссионного вентиляторного программирования», обеспечивающего этап вспомогательного раздутия (наполнения) легких, за которым следует перкуссионная дефляция легких до заданного экспираторного базового уровня (во время пассивного выдоха), обеспечивая тем самым:

- эндобронхиальное перкуссионное смешение;

- пошаговое раздутие - увеличение объема легких;

- автоматический стабилизатор (уровня) CPAP;

- интервал в конце вдоха способствует пассивному экспираторному удалению секреторных мacc.

Целью данного исследования явилось определение особенностей респираторной и интенсивной терапии пациентов с легочными инфекционными осложнениями на фоне острого и хронического отторжения трансплантата почки.

\section{Материал и методы}

Фундаментом исследования послужили результаты обследования и лечения 54 пациентов после ортотопической родственной ТП за период 2010-2017 гг. Из них 51 больной оперирован в нашем центре. Легочные осложнения с развитием двусторонней интерстициальной пневмонии наблюдались у 3 пациентов в ближайшем послеоперационном периоде, у 4 - в отдаленном, за период наблюдения от 1 месяца до 4 лет.

Еще 3 пациента, оперированные в клиниках Индии и Пакистана, были госпитализированы в наш центр с клиникой синдрома острого повреждения легких: в одном случае - на фоне острого и в двух - хронического отторжения трансплантата почки.

Критерии постановки диагноза основывались на регламентирующих документах Международной междисциплинарной классификации идиопатических интерстициальных пневмоний 2013 г. обществ American Thoracic Society и European Respiratory Society [6].

Использованы традиционные методы выделения и идентификации микроорганизмов, определения их чувствительности к антимикробным 
препаратам и динамический контроль содержания C-реактивного белка (СРБ). Видовую специфичность выделенных микроорганизмов определяли с помощью общепринятых методов с применением идентификационных сред (производство "HiMedia", Индия). Кроме того, определяли наличие антител против цитомегаловируса (ЦМВ) в сыворотке крови и ДНК ЦМВ количественным методом полимеразной цепной реакции (ПЦР).

После операции через 6-12 часов при возможности перорального приема препаратов реципиенты получали 3-компонентную поддерживающую иммуносупрессивную терапию, которую назначали согласно клиническим рекомендациям Научного общества и Ассоциации нефрологов России [7]. Реципиенты получали средние дозировки прографра (такролимуса) - 0,12-0,13 мг/ кг массы тела в сутки и селсепта (микофенолата мофетила) - 2 г/сут с последующим снижением до 1 г/сут. В качестве третьего компонента назначали невысокие дозировки кортикостероидных гормонов - не более 0,5 мг/кг массы тела больного. Кроме того, все пациенты получали противовирусную, антибактериальную, инфузионную и симптоматическую терапию.

Респираторная терапия заключалась в применении неинвазивной ИВЛ согласно протоколу ATS/ERS (NiCPAP ASB, PEEP - 8, Trigger 3 l/min, $\mathrm{FiO}_{2}-50 \%$ ) с перерывами, при которых использовали маску положительного давления в конце выдоха (ПДКВ) и ИПВЛ в режиме низкой и средней перкуссии в комбинации с небулайзерной терапией (стандартный протокол + раствор ФарГАЛСа в разведении $1: 4)$.

Для проведения NiCPAP применяли аппарат VELLA (Viasys Healthcare Inc.). Процедуры механической перкуссионной вентиляции легких выполняли при помощи аппарата IPV-HCBIPHASICIMPULSATOR (Percussionaire).

Так, применение NiCPAP и ИПВЛ основывалось на следующих моментах:

- неинвазивная ИВЛ с постоянным ПДКВ;

- мобилизация легочных дыхательных путей, проходимость которых была нарушена из-за задержки секреторных масс, отека слизистой и подслизистой оболочек, а также спазма бронхов;

- создание двусторонней равноценной альвеолярной вентиляции с целью улучшения поступления кислорода и удаления углекислого газа;

- механическое перемешивание внутрилегочных газов при помощи дифрфузной внутрилегочной перкуссии для улучшения эндобронхиальной дифрфузии кислорода и мобилизации углекислоты на периферии (легких);

- обеспечение существенного периодического «конвекционного дыхательного потока» для вымывания $\mathrm{CO}_{2}$.

\section{Результаты и обсуждение}

На фроне выраженного нарушения иммунного статуса пациентов и прогрессирования респираторного статуса при стандартном подходе к лечению были прогнозируемы интубация трахеи и перевод на традиционную пролонгированную ИВЛ с высоким риском присоединения ВАП. В этой связи был использован весь комплекс неинвазивной респираторной терапии с положительным результатом.

При оценке динамики газового состава артериальной крови отмечено значительное улучшение $\mathrm{SaO}_{2}, \mathrm{PaO}_{2}$ и $\mathrm{PaO}_{2} / \mathrm{FiO}_{2}$ по сравнению с исходными значениями (рис. 1). В отношении показателей альвеолярно-артериальной $\left(\mathrm{P}_{(\mathrm{A-a)}} \mathrm{O}_{2}\right)$ и артериально-альвеолярной разниц $\left(\mathrm{P}_{(\mathrm{a}-\mathrm{A})} \mathrm{O}_{2}\right)$ по кислороду (рис. 2), степени внутрилегочного шунтирования (Qs/Qt) также выявлена положительная динамика (рис. 3).

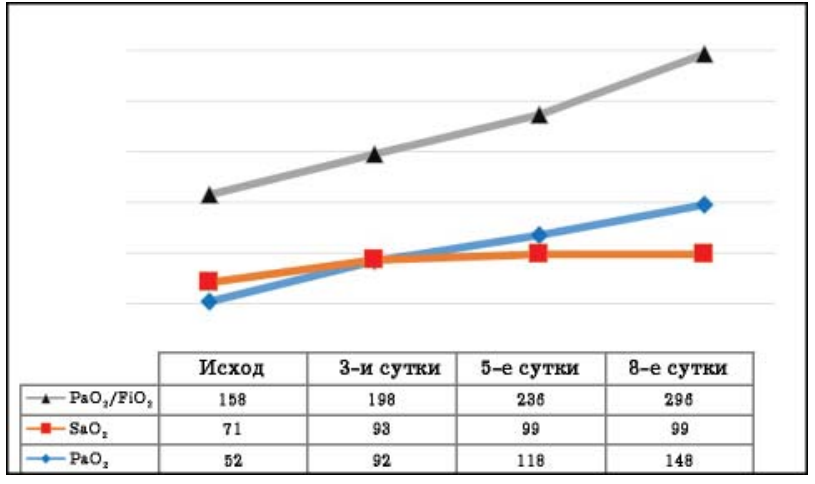

Рис. 1. Динамика газового состава артериальной крови

Fig. 1. Changes in arterial blood gas content over time

Длительность ИВЛ составила $9 \pm 2,6$ суток.

Приводим пример рентгеновских снимков пациента А., 36 лет, с диагнозом «двусторонняя интерстициальная пневмония, среднетяжелый ОРДС; хроническое отторжение трансплантата, состояние после гетеротопической трансплантации почки от живого родственного донора». Уже на 3-и сутки отмечался регресс клинических и рентгенологических проявлений ОРДС (рис. 4). 


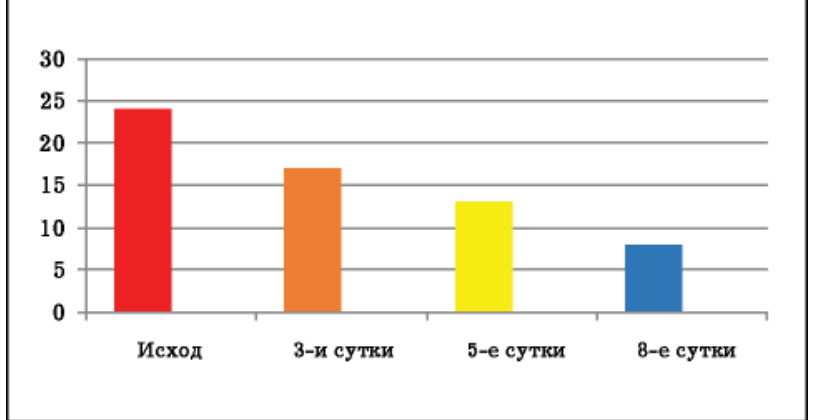

Рис. 2. Динамика альвеолярно-артериальной разницы $\left(\mathrm{P}_{(\mathrm{A-a)})} \mathrm{O}_{2}\right)$ по кислороду

Fig. 2 .Changes of alveolar-arterial $\left(P_{(A-a)} O_{2}\right)$ oxygen gradient over time

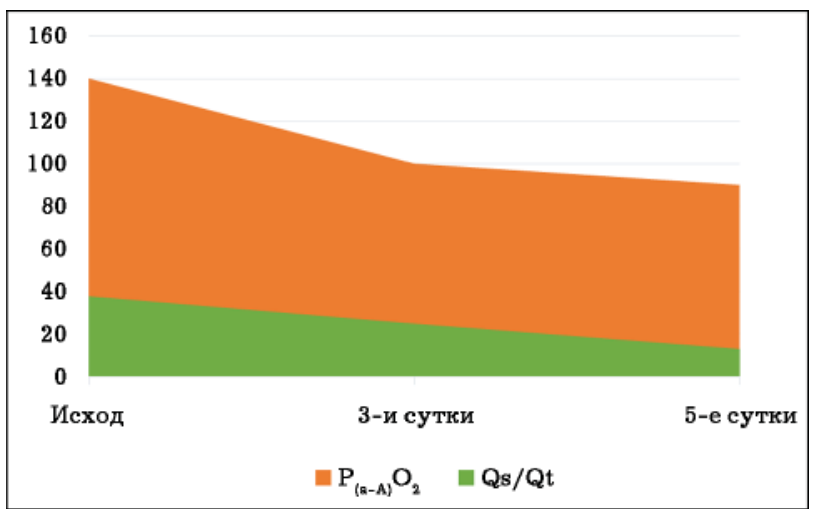

Рис. 3. Динамика артериально-альвеолярной разницы $\left(P_{(a-A)} \mathrm{O}_{2}\right)$ по кислороду и легочного шунта (Qs/Qt)

Fig. 3. Changes of and arterial-alveolar $\left(P_{(\mathrm{a}-\mathrm{A})} \mathrm{O}_{2}\right)$ oxygen gradient and pulmonary shunt $(\mathrm{Qs} / \mathrm{Qt})$ over time

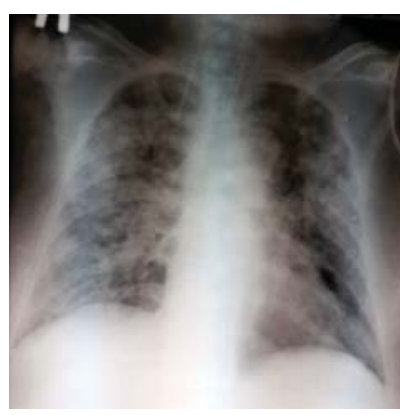

Исход

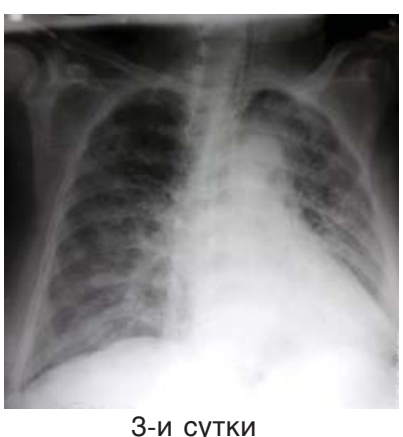

3-и сутки
Рис. 4. Динамика рентгенологической картины на этапах интенсивной терапии

Fig. 4. Changes in X-ray pattern at intensive care stages over time

У 3 пациентов появление отечно-асцитического синдрома и нарастание показателей мочевины и креатинина крови послужили поводом для их подключения к интенсивной терапии гемодиализом, который проводили ежедневно в тече- ние 3 часов с удалением в среднем до 1,5 литра ультрафильтрата. Одновременно с началом гемодиализа дозы такролимуса были снижены с 6 мг до 3 мг в сутки, а микофенолата мофетила повышены с 1000 мг до 2000 мг в сутки.

Выделенная микрофлора во всех случаях была представлена грамотрицательными микроорганизмами (E. coli, K. pneumoniae). Анализ антибиотикограмм показал, что в их отношении наиболее активными препаратами оказались пиперициллин/тазобактам, имипенем, цефоперазон / сульбактам и офрлоксацин. При показателях СРБ свыше 70 мг/л проводили деэскалационную антибактериальную терапию.

В связи с выявлением антител IgG против ЦМВ - ОП 3,0 нм (ОП кр 0,273 нм) была применена антивирусная терапия ганцикловиром, после которой контрольное определение качественной ПЦР было отрицательным.

В раннем послеоперационном периоде наблюдался 1 смертельный исход у 47-летнего мужчины. Причиной смерти явились криз отторжения, двусторонняя нижнедолевая пневмония, ОРДС тяжелой степени, острая сердечно-сосудистая недостаточность.

Выводы

1. Неинвазивные респираторные методики интрапульмональной перкуссионной вентиляции легких и NiCPAP (Noninvasive Constant Positive Airway Pressure) с включением гемодиализа являются альтернативной лечебной тактикой у пациентов с двусторонней интерстициальной пневмонией и интерстициальным отеком на фоне острого и хронического отторжения трансплантата почки.

2. Возможности вентиляционной технологии интрапульмональной перкуссионной вентиляции легких у больных с легочной патологией позволяют оптимизировать терапию, защитить дыхательные пути, избежать негативных последствий искусственной вентиляции легких. Интрапульмональная перкуссионная вентиляция легких может являться самостоятельным видом респираторной терапии, использующим оригинальный технический прием дыхания при помощи интермиттирующего положительного давления с созданием экстраторакальной перкуссии, и небулайзерной терапии; она наиболее эффективна при гиперсекреции слизи в просвет бронхиального дерева и необходимости дренирования мокроты. 


\section{АКТУАЛЬНЫЕ ВОПРОЯЫ НЛИНИЧЕНКОЙ ТРАНЯППАНТОЛОГИИ}

\section{AGTUAL ISSUES OF TRANSPLANTATION}

3. Комплексная профилактика и лечение гнойно-воспалительных заболеваний легких с применением небулайзерной терапии и фрибробронхоскопического бронхиального лаважа позволяют снизить частоту ранних и поздних специ- фических бронхолегочных осложнений, добиться клинического улучшения состояния больных уже на 2-е-3-и сутки и сократить сроки их выздоровления.

\section{КОНФЛИКТ ИНТЕРЕСОВ. АВТорЫ ЗаЯВЛЯЮт Об ОТсутствИИ КОНФЛЛИкта иНтереСОВ. CONFLICT OF INTERESTS. Authors declare no conflict of interest.}

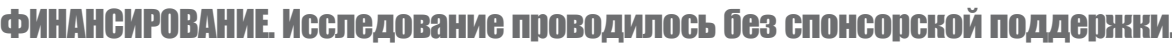 FINANCING. The study was performed without external funding.}

\section{Лит ература}

1. SarnakM.J., JaberB.L. Pulmonary infectious mortality among patients with endstage renal disease. Chest. 2001;120(6):1883-1887. PMID:11742917 2. Jha V., Sakhuja V., Gupta D., et al. Successful management of pulmonary tuberculosis in renal allograft recipients in a single center. Kidney Int. 1999;55(6):1944-50. PMID:10571806 DOI:10.1046/j.15231755.1999.00746.x
3. Maraha B., Bonten H., van Hoof H., et al. Infectious complications and antibiotic use in renal transplant recipients during a 1-year follow-up. Clin. Microbiol. Infect. 2001;7(11):619-625. PMID:11737086

4. Готье С.В. (ред.) Инфекции в трансплантологии. М., Тверь: Триада, 2010: 113.

5. Riffard G., Toussaint M. Intrapulmonary percussion ventilation: operation and settings. Rev. Mal. Respir. 2012;29(2):347-354. PMID:22405124 DOI:10.1016/j.rmr.2011.12.003

\section{References}

1. Sarnak M.J., Jaber B.L. Pulmonary infectious mortality among patients with endstage renal disease. Chest. 2001;120(6):1883-1887. PMID:11742917 2. Jha V., Sakhuja V., Gupta D., et al. Successful management of pulmonary tuberculosis in renal allograft recipients in a single center. Kidney Int. 1999;55(6):1944-50. PMID: 10571806 DOI:10.1046/j.15231755.1999.00746.x 3. Maraha B., Bonten H., van Hoof H., et al. Infectious complications and antibiotic use in renal transplant recipients during a 1-year follow-up. Clin Microbiol Infect. 2001;7(11):619-625. PMID:11737086

4. Gautier S.V., ed. Infections in transplantology. Moscow, Tver': Triada Publ., 2010. 113 p. (In Russian).

5. Riffard G., Toussaint M. Intrapulmonary percussion ventilation: operation and settings. Rev Mal Respir. 2012;29(2):347-354. PMID:22405124 DOI:10.1016/j.rmr.2011.12.003
6. Dellamonica J.,Vargas F. High frequency percussion ventilation: indications need to be more precisely defined. Rev. Mal. Respir. 2012;29(2):111113. PMID:22405104 DOI: $10.1016 / \mathrm{j}$. rmr.2011.06.019

7. Мойсюк Я.Г., Ватазин А.В., Прокопенко Е.И. и др. (рабочая группа). Проект клинических рекомендаций по диагностике и лечению болезней трансплантированной почки. 2014. 19 c. URL: health. admtyumen.ru>files...Клинические...Б... почки.pdf
6. Dellamonica J., Vargas F. High frequency percussion ventilation: indications need to be more precisely defined. Rev Mal Respir. 2012;29(2):111-113. PMID:22405104 DOI:10.1016/j. rmr.2011.06.019

7. Moysyuk Ya.G., Vatazin A.V., Prokopenko E.I., et al. (working group). Draft clinical recommendations for the diagnosis and treatment of diseases of the transplanted kidney. 2014. 19 p. Available at: health.admtyumen.rusfiles... Клинические...Б...почки.pdf 